\title{
Importance of Early and Multidisciplinary Intervention in Patients with Coexistent Juvenile Idiopathic Arthritis and Familial Mediterranean Fever
}

\author{
Selin ÖZEN, Haydar GÖK, Şehim KUTLAY \\ Department of Physical and Rehabilitation Medicine, Ankara University Faculty of Medicine, Ankara, Turkey
}

\begin{abstract}
Familial Mediterranean fever (FMF) and juvenile idiopathic arthritis (JIA) are childhood-associated diseases that are rarely found to coexist. Treatment includes non-steroidal anti-inflammatory drugs (NSAIDs), intra-articular steroid injections, immunosuppressants, increased range of motion and strength, and prevention and correction of deformities that require a multidisciplinary approach. Here, we describe the clinical presentation, radiological findings, and management strategies of a 19-year-old male with a diagnosis of JIA and FMF presenting with musculoskeletal deformities. Coexistence of JIA and FMF has a progressive nature and can result in severe joint erosions, development of joint contractures, muscle atrophy, and osteoporosis, despite medical treatment. A multidisciplinary approach including an early and intensive rehabilitation program with regular followup can be considered as an important adjunct to medical treatment. This in turn may reduce disability and improve quality of life.

Keywords: Juvenile idiopathic arthritis, familial Mediterranean fever, anti-tumor necrosis factor
\end{abstract}

\section{Introduction}

Juvenile idiopathic arthritis (JIA) is the most common chronic arthritic diseases occurring in childhood (1). Fifty percent of cases occur in children between 2 and 6 years of age (2). Some of the main clinical features of JIA include systemic arthritis, oligoarthritis rheumatoid factor, negative and positive polyarthritis, and enthesitis-related arthritis (3). JIA can result in contractures, pain, muscle atrophy, and osteoporosis, thereby resulting in limitations to daily activities of living. Therefore, following diagnosis, the main goal is the prevention of progression. In addition to receiving medical treatment, patients should be closely followed up by a physiatrist or a pediatric rheumatologist, and an early rehabilitation program should be initiated. It is important to educate the patient and family, maintain joint mobility, and attempt to prevent joint deformities from occurring.

Familial Mediterranean fever (FMF) is an autosomal recessive genetic disease mainly occurring in Ashkenazi Jewish, Armenian, Turkish, and Arab communities $(4,5)$. FMF is characterized by the attack of fever alongside abdominal, chest, or joint pain. Attacks of febrile myalgia and chronic arthritis occur less commonly (3). Furthermore, if poorly controlled, FMF results in the gradual development of AA-type nephropathic amyloidosis (2). FMF and JIA often involve the same population of children and may be confused at times. The coexistence of FMF and JIA is extremely rare with only a limited number of cases reported in the literature. It is often associated with erosive and progressive joint disease (6). Failing to initiate an early and intensive rehabilitation 
program along with medical treatment may result in severe disability. Herein, we report the case of a 19-year-old patient who had coexisting JIA and FMF and received regular drug treatment and was presented to a rehabilitation clinic at a later stage of disease with severe musculoskeletal deformities and disability.

\section{Case Report}

A 19-year-old male patient with a diagnosis of JIA and FMF presented with limitations in movement, joint pain, and difficulty in walking. A diagnosis of JIA was made at the age of 5 years after the swelling of the right ankle, followed by swelling of the left ankle, bilateral knees, wrists, and bilateral $2^{\text {nd }}, 3^{\text {rd }}$, and $4^{\text {th }}$ proximal interphalangeal joints. There were no pathological eye findings. The patient was admitted for treatment by the Cardiology team and initially followed up by Paediatric Cardiology Department and then by Paediatric Nephrology Department as an outpatient. The patient and five of his siblings were diagnosed with FMF in the year 2000 after symptoms of fever accompanied by abdominal pain and pleuritic chest pain and detection of the MEFV gene mutation, and the gene mutation was associated with the FMF phenotype. His drug regime included $15 \mathrm{mg}$ methotrexate once weekly (since 1996), $0.8 \mathrm{mg} /$ kg etanercept weekly (since 1999), non-steroidal anti-inflammatory drugs (NSAIDs), $1.5 \mathrm{mg}$ colchicine once daily, and folic acid supplementation.

In December 2011, the patient was admitted to the Paediatric Nephrology Clinic for reorganisation of his medical treatment. He was found to be anemic with markedly increased erythrocyte sedimentation rate (ESR) and C-reactive protein (CRP) levels. Sacroiliac magnetic resonance imaging (MRI) revealed pathological signals from the head of the femur bilaterally. Cervical, thoracic, and lomber MRI findings were normal. Because of his widespread musculoskeletal complaints, the patient was referred to our Rehabilitation Clinic and admitted for treatment.

Examination of the respiratory, cardiovascular, and gastrointestinal systems were normal. On his musculoskeletal examination, active and passive right shoulder flexion and abduction were reduced to $100^{\circ}$. Active and passive left shoulder flexion and abduction were reduced to $90^{\circ}$. There was a $5^{\circ}$ loss of left elbow extension. Bilateral wrist extension was reduced and painful on movement. Nodules were present on the proximal interphalangeal joints. On examination of the vertebral column, there was an increase in thoracic kyphosis and lumbar lordosis (Figure 1). Anterior pelvic tilt was present. Low back range of motion (ROM) was restricted in all planes, with a modified Schober of $4.5 \mathrm{~cm}$. Bilateral hip flexion was reduced to $40^{\circ}$ on passive and active movement with shortening of the hamstrings. The right knee was swollen with a positive patellar shock. The left knee had a $5^{\circ}$ flexion contracture. Bilateral knee flexion was reduced and painful. Ankle and foot ROM was normal but painful. Neurological examination revealed normal tone of all extremities, normal power, sensation, and coordination. Deep tendon reflexes were normal. On examination of his gait, the patient walked with his hips and knees flexed, making initial contact with his toes. Blood tests revealed an ESR of $22 \mathrm{~mm} / \mathrm{h}$

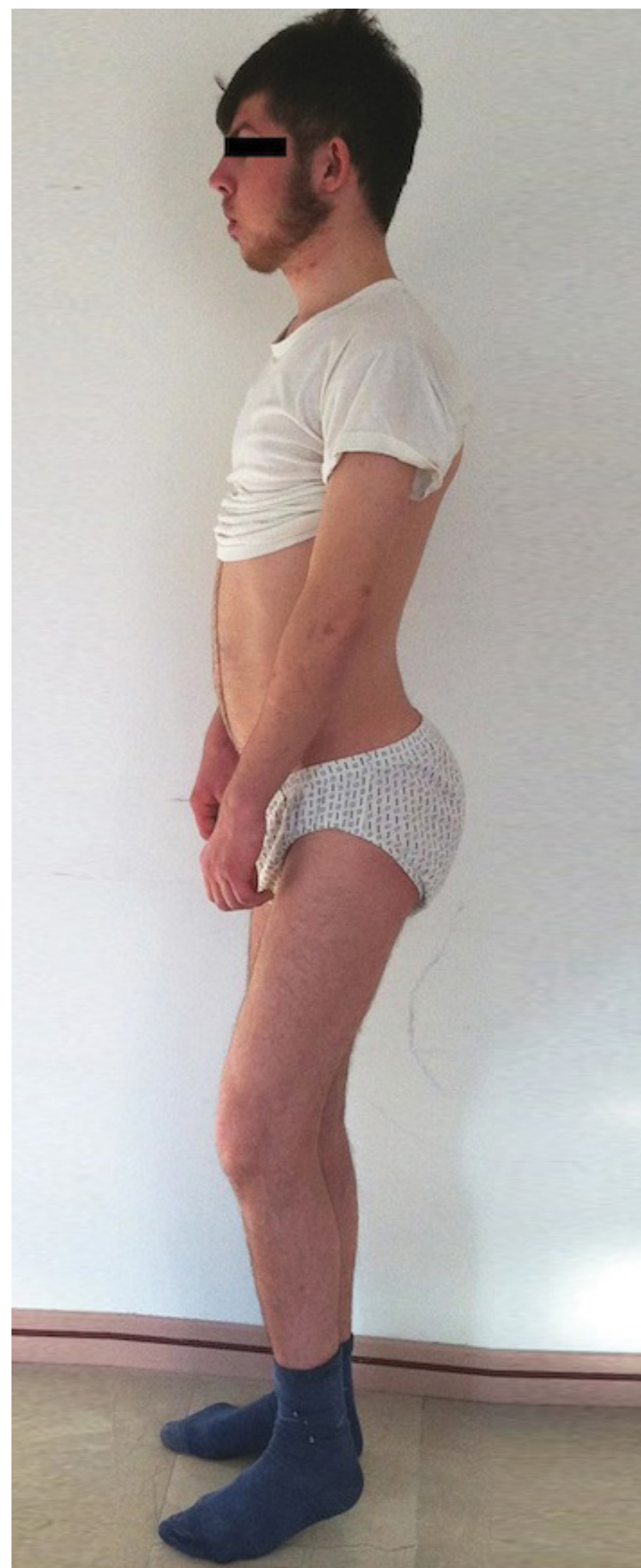

Figure 1. Image showing the side profile of the patient's posture 


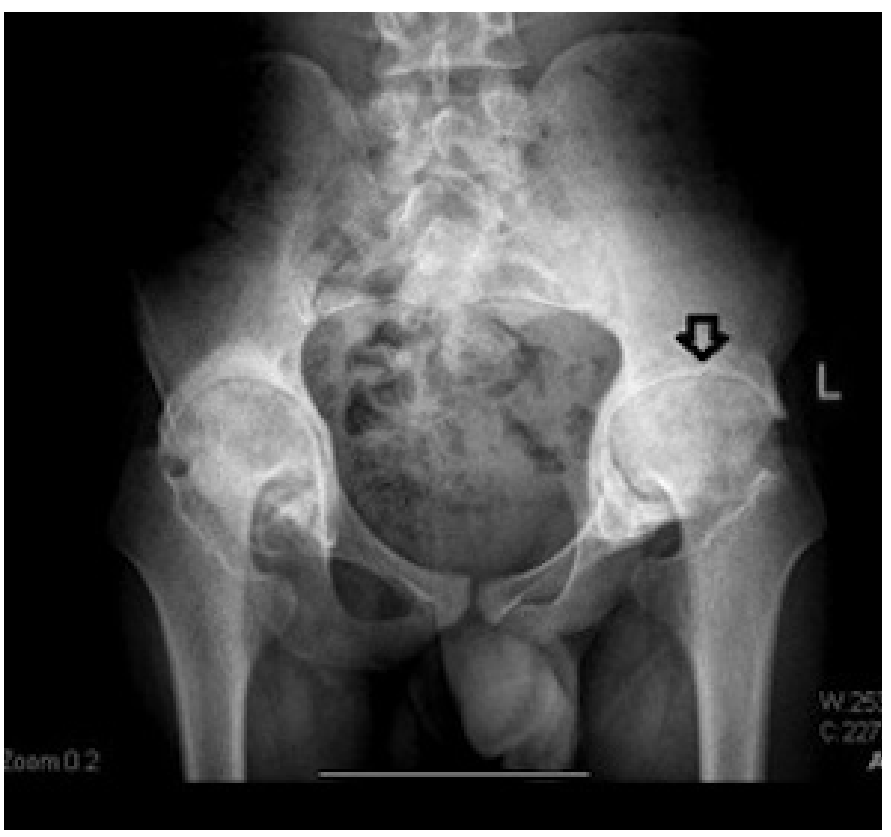

Figure 2. Bilateral protrusio acetabuli and sclerosis of the hip joint

and increased CRP (21.1 mg/L). Antinuclear antibody (ANA) was found to be positive (previously negative in 2006). Human leukocyte antigen B27 (HLA B27) and rheumatoid factor were negative. Protein electrophoresis showed an increase in the serum albumin (51.7\%) and gamma globulin (23.2\%) bands. He had proteinuria $(200 \mathrm{mg} / 24 \mathrm{~h})$. Pelvic X-ray revealed bilateral protrusio acetabuli, extensive narrowing and sclerosis of the hip joints, and osteoporosis (Figure 2). Bilateral hand X-rays revealed band-like osteoporosis. Knee X-rays revealed bilateral joint space narrowing with accentuated intercondylar eminences.

The physical therapy program included the following: electrotherapy as well as lower extremity stretching and strengthening exercises for limited joints. Bike riding was attempted but was unsuccessful because of the limited right hip flexion. In response to the treatment, his joint pain reduced. The right knee swelling resolved and bilateral knee flexion marginally increased. The vertebral column deformity as well as hip and knee contractures remained. Bilateral hip arthroplasty was recommended; however, the patient declined surgery. At the end of the rehabilitation program, consent for the presentation of his case was obtained from the patient prior to his discharge.

\section{Discussion}

This case of JIA coexisting with FMF presenting with severe musculoskeletal involvement highlights the importance of early and intensive rehabilitation. Even when clinical remission is achieved using drug therapy to inhibit the formation of musculoskeletal deformities, rehabilitation must be implemented at an early stage of disease and at frequent intervals.

Rheumatoid negative polyarthritis is the most heterogeneous subtype of JIA. The findings in this case are compatible with the JIA subtype characterised by symmetric polyarthritis of the large and small joints. This subtype usually starts at school age; ANA is negative and has a variable outcome (3). As seen in this case, MEFV gene mutations may be associated with coexistent FMF and JIA. These gene mutations may contribute to the inflammatory process, thereby increasing disease progression (6). In this case, rapid progression was prominent despite extensive treatment with disease-modifying antirheumatic drugs (DMARDs) and anti-tumor necrosis factor (anti-TNF) drugs. On admission, the patient's ANA was positive, whereas it had previously been negative. ANA positivity is not associated with FMF (7). However, ANA positivity has been reported as positive in $11 \%$ of anti-TNF users, and we believe that this is the case in our patient (8).

Rozenbaum et al. (6) described three cases of JIA coexisting with FMF. In the first case, the patient was diagnosed with JIA at the age of 8 years. Similar to our case, the patient first developed bony erosions in both hands. He went onto develop bilateral knee osteonecrosis and subsequently required bilateral knee replacements at the age of 31 years. In the second case, a diagnosis of JIA was made in a 7-year-old patient following the development of persistent pain in the wrists, hands, and knees. The hand deformities that ensued secondary to arthritis were more severe than those seen in our case; she developed swan neck deformities with erosive subluxation of the hands and ulnar deviation. As in our patient, end-stage osteoarthritis of the hips developed, and total hip arthroplasty of the right hip followed by total hip arthroplasty of the left hip were performed at ages of 25 years and 30 years, respectively. In the third case, once again, following a complaint of persistent joint pain at the age of 16 years, JIA with severe erosive polyarthritis of hands and feet was diagnosed. Bilateral osteonecrosis of the femoral heads resulted in hip replacement in the patient at the age of 32 years. As in our case, the latter two patients developed persistent proteinuria and all had a previous diagnosis of FMF.

To date, few cases of coexisting FMF and JIA have been reported, and all had a poor prognosis, eventually requiring joint replacement because of extensive bone erosions and musculoskeletal deformities (6). Similarly, in this case, despite the patient's young age, total hip arthroplasty was considered as a treatment option. The cases reported in the literature were all managed with drug therapy alone, and none received physical therapy or rehabilitation. On the other hand, a rehabilitation program was ensued at a later and advanced stage of disease in this case.

\section{Conclusion}

Due to the unfavorable disease course of JIA coexisting with FMF, an early and intensive rehabilitation program may protect joint motion and improve mobility, thereby preventing the occurrence of or curbing secondary musculoskeletal deformities in young patients who would otherwise develop severe disability.

Informed Consent: Written and verbal informed consent was obtained from the patient on whom this case report is based.

Peer-review: Externally peer-reviewed. 
Author Contributions: Concept - S.Ö., Ş.K.; Design - S.Ö., H.G.; Supervision - Ş.K., H.G.; Data Collection and/or Processing - S.Ö.; Analysis and/or Interpretation - Ş.K., H.G.; Literature Review - S.Ö.; Writer - S.Ö., H.G.; Critical Review - Ş.K., H.G.

Conflict of Interest: No conflict of interest was declared by the authors.

Financial Disclosure: The authors declared that this study has received no financial support.

\section{References}

1. Petty RE, Southwood TR, Baum J, Bhettay E, Glass DN, Manners P, et al. Revision of the proposed classification criteria for Juvenile Idiopathic Arthritis; Durban, 1997. J Rheumatol 1998;25:1991-4.

2. Rietschel C, Latta K. Rheumatic joint disease in childhood and adolescence. Orthopade 2012;41:227-40. [CrossRef]

3. Ravelli A, Martini A. Juvenile idiopathic arthritis. Lancet 2007;369:767-78. [CrossRef]
4. Sohar E, Gafni J, Pras M, Heller H. Familial Mediterranean Fever. A survey of 470 cases and review of the literature. Am J Med 1967;43:227-53. [CrossRef]

5. Daniels M, Shohat T, Brenner-Ullman A, Shohat M. Familial Mediterranean Fever: High gene frequency among the non-Ashkenazic and Ashkenazic Jewish population in Israel. Am J Med Genet 1995;55:311-4. [CrossRef]

6. Rozenbaum M, Rosner I. Severe outcome of juvenile idiopathic arthritis (IIA) associated with familial Mediterranean fever (FMF). Clin Exp Rheumatol 2004;22:75-8.

7. Güler E, Kaptanoğlu E, Şahin O, Candan F, Hayta E, Elden H. Autoantibodies are not associated with familial mediterranean fever. Acta Reumatol Port 2012;37:144-8.

8. Cush JJ: Cytokine inhibitors.in Hochberg MC, Silman AJ, Smolen JS, Weınblatt ME, Weisman MH (eds.): Rheumatology. 3rd ed., Edinburg, Mosby, 2003;461-84. 